\title{
Selection of Transfected Mammalian Cells
}

\author{
Richard M. Mortensen ${ }^{1}$ and Robert E. Kingston ${ }^{2}$ \\ ${ }^{1}$ University of Michigan, Ann Arbor, Michigan \\ ${ }^{2}$ Massachusetts General Hospital and Harvard Medical School, Boston, Massachusetts
}

\section{ABSTRACT}

To determine the function of a gene in vitro, expression in heterologous cells is often employed. This can be done by transient expression, but often requires a more permanent expression of the gene and the creation of a cell line. This process can involve decisions as to the nature of construct used for expression, and invariably uses some strategy to select the transfected cells. Typically, these strategies use one of a number of genes that confer resistance to an added drug that will kill untransfected cells but not the transfected cells (positive selection). Alternatively, sometimes the strategy uses a gene that will confer sensitivity to a compound and kills the transfected cells (negative selection). This chapter discusses some of the strategies and genes used in creating cell line for in vitro study of gene function. Curr. Protoc. Mol. Biol. 86:9.5.1-9.5.13. (c) 2009 by John Wiley \& Sons, Inc.

Keywords: stable integration • selection • mammalian cell • stable transfection • selection marker $\bullet$ Cre-lox $\bullet$ Flp-FRT $\bullet$ expression

\section{INTRODUCTION}

Analysis of gene function frequently requires the formation of mammalian cell lines that contain the studied gene in a stably integrated form (Chapter 16). Approximately one in $10^{4}$ cells in a transfection will stably integrate DNA (the efficiency can vary depending on the cell type). Therefore, a dominant, selectable marker is used to permit isolation of stable transfectants. In the first part of this unit, the procedures for determining selection conditions and for the resulting stable transfection are presented (see Basic Protocol 1) and the most commonly used selectable markers are discussed (see Basic Protocol 2). Basic Protocol 2 includes conditions for thirteen markers commonly used for selection of mammalian cells.

In order to study the function of a particular gene, it is often necessary to work with a homogeneous population of cells overexpressing either wild-type or mutant variants of the corresponding protein. Two points must be considered, however, when designing such experiments. First, if the gene product of interest has a negative or toxic effect on cell growth, selection of cells stably expressing that gene cannot be achieved using a dominant selection marker. Cells carrying an antibiotic-resistance marker lose their growth advantage when expressing a toxic gene. Second, isolation of a homogeneous pool of cells stably expressing a gene by antibiotic selection often takes a month or more. By the time a stably transfected pool of cells is isolated, many important, temporally dependent biochemical events may have already occurred.

\section{STRATEGIC PLANNING}

There are several aspects of the vector to be considered for use in stable transfection. The following parameters should be taken into account when designing vectors to express either the marker gene or a gene of interest that is introduced with the marker gene.

\section{Construct Design}

A typical construct is depicted in Figure 9.5.1.
Introduction of

DNA into

Mammalian

Cells

9.5.1

Supplement 86 


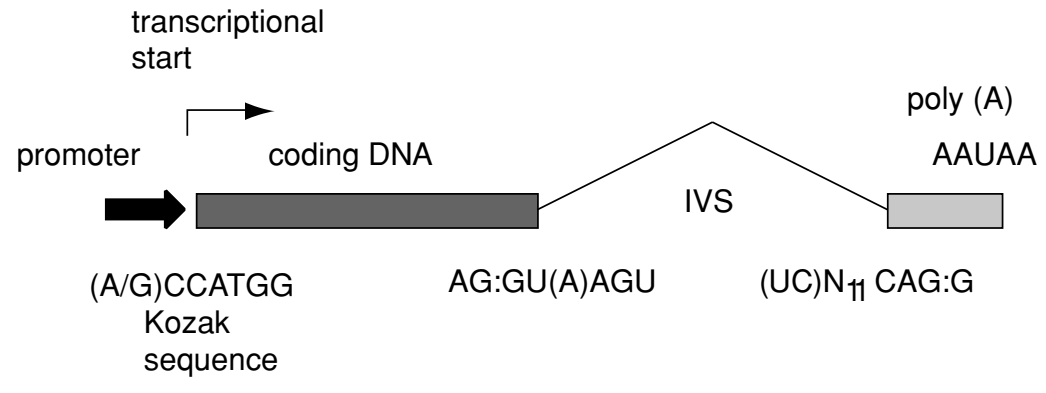

Figure 9.5.1 Generalized construct for expression of genes in mammalian cells. Each integrated construct must contain a promoter region with a transcriptional start site and a coding sequence. Features that increase expression include translational start sites according to Kozak's rules, intervening sequences with splice donor and acceptor sites, and polyadenylation signals. Abbreviation: IVS; intervening sequence.

\section{Promoters}

The choice of promoter can be critical for efficient expression, as it is the promoter and accompanying enhancers that drive the expression of the coding regions of the gene of interest and the selectable marker. Promoters are usually short DNA sequences $(<1 \mathrm{~kb})$ that bind endogenous transcription factors. They may or may not have a TATA box. Cytomegalovirus and simian virus 40 (SV40) promoters have been favorite general mammalian promoters for years, and many derivatives have been made and are commercially available. More recently, other promoters such as pMC1 and PGK (promoter from phosphoglycerate kinase) have been popular because of the high levels of expression obtainable. It is also possible to use a promoter that is specific for the cell type.

Conditional-expression promoters have also been introduced recently, increasing the utility and flexibility of stably transfected lines. These promoters require the introduction of a specific transactivator, which is sensitive to an administered compound. Two transcription factors have been developed, based on the tet operon, which use tetracycline to turn expression off (tet-off) or on (tet-on). For details of tetracycline-regulated systems, see UNIT 16.14, Gossen and Bujard (1992), and Gossen et al. (1995). In addition, the insect hormone ecdysone has been used to activate expression based on the ecdysone receptor and responsive element (No et al., 1996). Many versions of these are available from commercial sources (e.g., Stratagene and Invitrogen).

\section{Translational start site}

The translational start site can dramatically affect expression and should be considered both in designing the expression construct for the gene of interest and in choosing the specific version of a selectable marker. Most selectable markers are based on a bacterial resistance gene, and in their native state may give low levels of expression. One major reason for this is the differences in translational start sites. Whereas bacterial systems translate polycistronic messages well, translation of most mammalian mRNA is greatly decreased $(\sim 10$-fold) by upstream ATG start sites. In addition, the mammalian start site is most efficient if it follows "Kozak's rules" (Kozak, 1989). Many commonly used selectable markers have been modified to give good mammalian translation.

\section{Polyadenylation site}

A sequence in the $3^{\prime}$ end of the expression vector that signals the addition of a poly(A)

Selection of Transfected Mammalian Cells tail often stabilizes the mRNA and leads to better expression. The polyadenylation signal consists of a conserved sequence (AAUAA) 11 to 30 nucleotides upstream of the 
polyadenylation site, and a GU- or U-rich region downstream. Many different sources of polyadenylation signals are used. A common one is derived from the SV40 virus, but constructs using the PGK promoter often use the phosphoglycerate kinase polyadenylation signal as well.

\section{Intervening sequence and splice sites}

Addition of an intervening sequence (intron) that is spliced out in the final mRNA can also increase expression. A $5^{\prime}$ and $3^{\prime}$ splice site flanking the intron are necessary for splicing out of the intron. The mechanism by which addition of an intron might increase expression is not well understood.

\section{Introduction of DNA into Cells}

For the creation of stable lines, the results can vary depending on the method of DNA introduction used. Calcium phosphate (UNIT 9.1) and dextran (UNIT 9.2), as well as liposomemediated and positively charged liposome-mediated (UNIT 9.4) transfection methods, all tend to introduce multiple copies of transfected genes, usually at a single site. These methods aggregate the DNA and are suitable for cotransfection of separate plasmids, typically in the ratio of 5 to 10 parts DNA of interest to one part DNA of selectable marker. These multiple copies can integrate as head-to-head, head-to-tail, or a combination of the two orientations. Because of the tandem array of similar sequences, intrachromosomal rearrangement can occur, leading to loss of copies and a decrease in the level of expression.

Electroporation (UNIT 9.3) will usually introduce DNA in a single copy in a single site, depending on the DNA concentration (i.e., high DNA concentrations will introduce multiple copies at a single site in $\sim 25 \%$ of cells, although $75 \%$ will still contain single copies). If a single copy is desired (as for removal of selection markers as described below), electroporation of colinear constructs is required.

Separate plasmids for the gene of interest and the selectable marker can be used if multiple copies of genes are desired. Both aggregation methods and electroporation will yield resistant colonies with multiple copies of the gene. If only a single copy is desired, then electroporation should be used with a single plasmid that contains both the gene of interest and the selectable marker.

\section{Removal of Selectable Markers}

The selectable marker can be removed using recombinases (Fig. 9.5.2). This is desirable if more than one sequential genetic manipulation is planned. Because tandem repeats would be eliminated by the recombinase expression, single-copy integration is desirable; therefore the preferred method of transfection is electroporation. Removal of the selectable marker is accomplished by transiently expressing the Cre recombinase, which removes the sequence between the identical recognition sequences (lox sites).

\section{Amplification of Expressed Gene}

Some selectable markers (most notably DHFR) can be used to amplify the expression of the integrated DNA. Amplification is accomplished by gradually increasing the selection pressure with higher concentrations of selection medium. This results in tandem duplication of the integrated DNA (see UNIT 23.5).

\section{Introduction of DNA at Specific Sites}

One of the problems with production of stable lines is that the transfected DNA integrates at a random site. Many of the integrations will not give adequate expression. To address this problem, two different approaches can be taken.

Introduction of

DNA into

Mammalian

Cells

9.5.3 


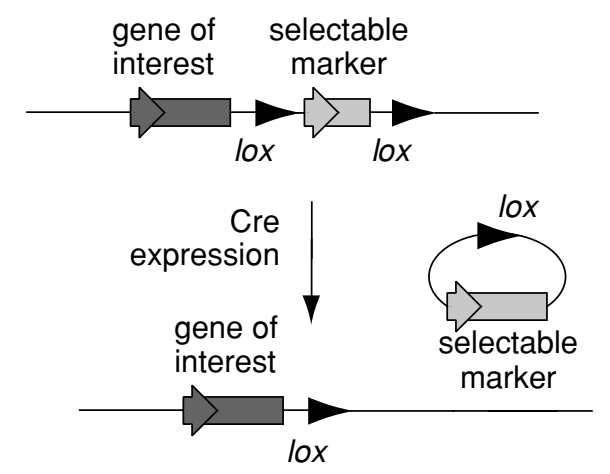

Figure 9.5.2 Using the Cre-lox system to remove a selectable marker after integration at a unique site. Transient expression of Cre recombinase causes the excision of sequences between the lox sites.

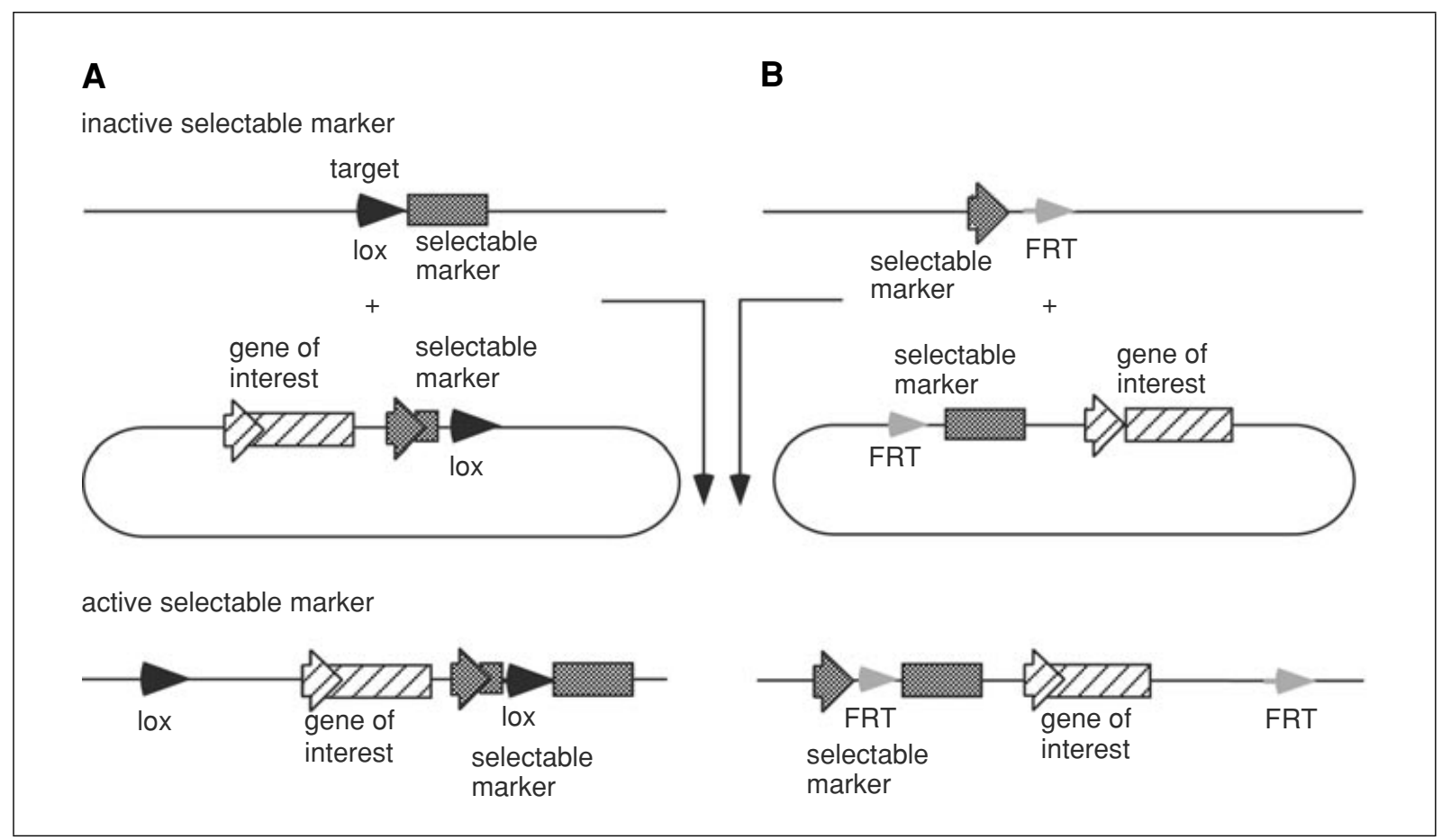

Figure 9.5.3 Using the Cre-lox or FIp-FRT systems to integrate and expression construct at a specific reproducible site. (A) The target site is a previously integrated defective form of a selective marker (lacking a promoter and ATG start codon). Transfection with a plasmid containing the gene of interest leads to integration at the lox site and adds a promoter and translational start site, restoring expression of the selectable marker. (B) The target site has an active promoter and the plasmid has the remainder of the selectable marker. Recombination with Flp results in active selectable marker.

Selection of Transfected Mammalian Cells
It is possible to use homologous recombination to introduce DNA at a specific site. While this approach has largely been applied to transgenic animals, the same principles can be applied to a cell line. It is possible then to create a cell line where the gene of interest is expressed by an endogenous promoter in its normal genomic location.

Sauer and co-workers developed the Cre recombinase system to introduce DNA at a selected site, shown in Figure 9.5.3 (Fukushige and Sauer, 1992). Initially an inactive modified neo is integrated into the DNA, which contains an in-frame loxP site at the $5^{\prime}$ end of neo without an ATG initiation codon or a promoter. This cell line can then be

\subsection{4}


transfected with a plasmid containing the gene of interest and a promoter in front of a modified lox site. When electroporated into the previously established cell line with a Cre expression vector, recombination is targeted to the loxP site in the genome and can be selected in G418 because of the reconstituted neo activity. This constant-site integration gives consistent expression of the gene of interest in different clones. A similar system using the Flp-FRT system is also marketed by Invitrogen (Flp-In System).

\section{STABLE TRANSFER OF GENES INTO MAMMALIAN CELLS}

In this protocol, appropriate selection conditions for the parental cell line are first determined. The gene to be studied is then cotransfected into that cell line with a gene that expresses a selectable marker. The cells are allowed to grow under selection for approximately ten doublings before individual colonies are picked and expanded into cell lines.

\section{Materials}

Complete medium (APPENDIX 3F)

Selective medium (see Basic Protocol 2)

Cloning cylinders (see recipe; also see Fig. 16.23.2)

Additional reagents and equipment for mammalian cell culture and counting cells (APPENDIX 3F) and transfection (UNITS 9.1, 9.3, \& 9.4)

NOTE: All reagents and equipment coming into contact with live cells must be sterile, and aseptic technique should be used accordingly.

NOTE: All culture incubations are performed in a humidified $37^{\circ} \mathrm{C}, 5 \% \mathrm{CO}_{2}$ incubator unless otherwise specified.

1. Ensure that the cell line to be transfected can grow as an isolated colony. For adherent cells, plate $\sim 100$ cells on a 10 -cm tissue culture dish and feed every 4 days for 10 to 12 days. Count the number of viable colonies (see APPENDIX $3 F$ for basic cell culture techniques).

Selection of stably transfected colonies requires that cells be grown essentially in isolation. A cell line that does not grow as an isolated cell will not be able to be stably transfected. The simplest way to determine how many colonies are present on a tissue culture dish is to stain with methylene blue. Aspirate off the medium, then place $\sim 2 \mathrm{ml}$ of $2 \%$ methylene blue (made up in 50\% ethanol) on the dish. Wait 2 min, pour off methylene blue, and wash residual dye off in a bucket of cold water.

Cell lines that survive poorly as single cells may be selected by using a feeder layer-i.e., a monolayer of cells treated to prevent cell division (Robertson, 1987).

2. Determine the selection conditions for the parental cell line. For example, if hygromycin-B selection is to be used, determine the minimum level of hygromycin-B that must be added to the medium to prevent cell growth. Split a confluent dish of cells 1:15 into medium containing various levels of the drug. Incubate the cells for 10 days, feeding with the appropriate selective medium every 4 days, and examine the dishes for viable cells.

Mammalian cells can divide once or twice under selective conditions that will eventually kill them. For example, DHFR-deficient cells will divide in selective medium until they deplete their endogenous reserve of nucleotides. For this reason, it is essential to split cells at least 1:15 when testing selection conditions, or the cells may reach confluence before the selection can take effect. Several selection markers commonly used in mammalian cell culture are described below. The choice of a marker is determined both by the cell type to be transfected and the reason for doing the transfection (see Basic Protocol 2).
BASIC

PROTOCOL 1

Introduction of

DNA into

Mammalian

Cells

9.5.5

Supplement 86 
3. Determine the most efficient means of transfecting the parental cell type (introduction to Section I).

Calcium phosphate- and liposome-mediated transfection (UNITS $9.1 \& 9.4$ ) and electroporation (UNIT 9.3) are the methods of choice for producing stable transfectants, as both techniques introduce a large amount of DNA into cells that pick up DNA. The presence of a large amount of DNA increases the probability that some of it will become stably integrated. Transfection mediated by DEAE-dextran does not work well for production of stable cell lines.

4. If calcium phosphate transfection is used, split the parental cell line 1:15 into complete medium the day before the DNA is applied to the cell. If electroporation is used, refer to UNIT 9.3, Basic Protocol 1, step 7. If liposome-mediated transfection is used, see UNIT 9.4.

5. Cotransfect desired gene and marker gene into parental cell line. Use a molar ratio of 5:1 (plasmid containing the gene of interest to plasmid containing the selective marker). Include a control where carrier DNA (e.g., pUC13) is used instead of the plasmid containing the gene to be studied. Do several separate transfections containing the gene of interest in case one of the transfections fails.

It is not necessary to physically link the selective marker to the gene of interest prior to transfection if a 5:1 ratio is used. If selection requires transfection of a large amount of the selection plasmid, then a 5:1 ratio will not be achievable in some cases, and a single plasmid should be constructed that contains both the selective marker and the gene of interest. If no colonies result from the transfection containing the gene of interest, but colonies do appear in the control containing pUC13, then the gene of interest may be cytotoxic.

Efficient transfection by electroporation requires linearization of the construct.

6. Allow the cells to double twice under nonselective conditions. Split the cells 1:15 into selective medium.

7. Place at least five dishes into selective medium from each transfected dish to maximize the number of colonies that can be picked and expanded into cell lines. Feed the cells with selective medium every 4 days (or as needed). After 10 to 12 days, inspect the plates for colonies by holding the plates up to a light at an angle.

One of the plates can be stained (see annotation to step 1) to facilitate counting the colonies. Note that staining kills the cells.

8. Pick large, healthy colonies.

Colonies should contain $\sim 500$ to 1000 cells when they are picked.

In some cases, it may be wise to continue selection after picking. Some large colonies can "protect" nonresistant cells.

BASIC PROTOCOL 2

Selection of Transfected Mammalian Cells

9.5.6

\section{SELECTABLE MARKERS FOR MAMMALIAN CELLS}

For many of the selection conditions outlined below, the desired concentration of one or more of the drugs used for selection will vary depending on susceptibility of a particular cell line to the drug. For these drugs, approximate ranges needed for selection are given. Precise ranges for the parental cell line to be used can be determined by characterizing cell viability across a range of concentrations of the drug (see Basic Protocol 1, step 2).

\section{Positive Selection}

\section{Adenosine deaminase (ADA)}

Selection conditions. Medium supplemented with $10 \mu \mathrm{g} / \mathrm{ml}$ thymidine, $15 \mu \mathrm{g} / \mathrm{ml}$ hypoxanthine, $4 \mu \mathrm{M}$ 9- $\beta$-D-xylofuranosyl adenine (Xyl-A), and 0.01 to $0.3 \mu \mathrm{M}$ 
2'-deoxycoformycin (dCF). Fetal bovine serum (FBS) contains low levels of ADA which will detoxify the medium, so serum should be added immediately prior to use.

Basis for selection. Xyl-A can be converted to Xyl-ATP and incorporated into nucleic acids, resulting in cell death. Xyl-A is detoxified to its inosine derivative by ADA. dCF is a transition state analog inhibitor of ADA, and is needed to inactivate ADA endogenous to the parental cell type. As the level of endogenous ADA varies with cell type, the appropriate concentration of $\mathrm{dCF}$ for selection will vary as well.

Comments. High levels of expression of the transfected ADA gene will be necessary to achieve selection in cells with high endogenous ADA levels. ADA-deficient CHO cells are available. ADA can be used in amplification systems (Kaufman et al., 1986), as increasing the level of $\mathrm{dCF}$ selects for cells that have amplified the ADA gene.

Reference. Kaufman et al. (1986).

\section{Aminoglycoside phosphotransferase (neo, G418, APH)}

Selection conditions. 100 to $800 \mu \mathrm{g} / \mathrm{ml}$ G418 in complete medium. G418 should be prepared in a highly buffered solution (e.g., $100 \mathrm{mM}$ HEPES, $\mathrm{pH} 7.3$ ) so that addition of the drug does not alter the $\mathrm{pH}$ of the medium.

Basis for selection. G418 blocks protein synthesis in mammalian cells by interfering with ribosomal function. It is an aminoglycoside, similar in structure to neomycin, gentamycin, and kanamycin. Expression of the bacterial APH gene (derived from tn5) in mammalian cells therefore results in detoxification of G418.

Comments. Varying concentrations of G418 should be tested, as cells differ in their susceptibility to killing by G418. Different lots of G418 can have different potencies, causing many investigators to buy a large amount of one lot to standardize selection conditions. Cells will divide once or twice in the presence of lethal doses of G418, so the effects of the drug take several days to become apparent.

Reference. Southern and Berg (1982).

\section{Bleomycin (phleo, bleo, zeocin)}

Selection conditions. Complete medium supplemented with 0.1 to $50 \mu \mathrm{g} / \mathrm{ml}$ phleomycin.

Basis for selection. This gene encodes a 13,000-Da protein that stoichiometrically binds the drug (bleomycin, phleomycin, or zeocin) and inactivates it.

Comments. Three homologous genes that confer bleomycin resistance have been isolated from the Gram-positive bacterial plasmid pUB110, from the central region of the Gramnegative transposon Tn5, and from resistant strains of Actinomycetes.

References. Mulsant et al. (1988); Sugiyama et al. (1994).

\section{Cytosine deaminase (CDA, CD)}

Selection conditions. Medium containing $1 \mathrm{mMN} N$-(phosphonacetyl)-L-aspartate, $1 \mathrm{mg} / \mathrm{ml}$ inosine, and $1 \mathrm{mM}$ cytosine.

Basis for selection. Cytosine deaminase converts cytosine to uracil. $N$-(phosphonacetyl)L-aspartate blocks the de novo synthesis pathway of pyrimidines and forces the cells to rely on a cytosine deaminase scavenger pathway.

Comment. Originally defined as a negative selectable marker.

Reference. Wei and Huber (1996).

Introduction of

DNA into

Mammalian

Cells

9.5.7 


\section{Dihydrofolate reductase (DHFR)}

Selection conditions. $\alpha^{-}$medium supplemented with 0.01 to $300 \mu \mathrm{M}$ methotrexate (MTX) and dialyzed fetal bovine serum (FBS).

Basis for selection. DHFR is necessary for purine biosynthesis. In the absence of exogenous purines, this enzyme is required for growth. Dialysis of serum to remove endogenous nucleosides and use of media devoid of nucleosides is therefore necessary for selection. MTX is a potent competitive inhibitor of DHFR, so increasing MTX concentration selects for cells that express increased levels of DHFR.

Comments. Extremely high levels of expression of the transfected normal DHFR gene are needed for selection in cell lines with high endogenous DHFR levels. A mutant DHFR gene is available that encodes an enzyme resistant to MTX (Simonsen and Levinson, 1983). This gene can be used for dominant selection in most cell types. DHFR can be used to amplify transfected genes. This is most efficiently accomplished using a DHFR-deficient CHO cell line and a normal DHFR gene for selection.

Reference. Simonsen and Levinson (1983).

\section{Histidinol dehydrogenase (hisD)}

Selection conditions. Complete medium supplemented with $2.5 \mathrm{mM}$ histidinol or medium lacking histidine and containing $0.125 \mathrm{mM}$ histidinol.

Basis for selection. This gene encodes an enzyme that catalyzes the oxidation of L-histidinol to L-histidine. Thus, medium deficient in histidine but containing histidinol will not support cell growth. In addition, histidinol is toxic in the presence of histidine because of inhibition of histidyl-tRNA synthase, so hisD-containing cells can be selected by their ability to detoxify histidinol.

Comment. Histidinol in complete medium usually works well.

Reference. Hartman and Mulligan (1988).

\section{Hygromycin-B-phosphotransferase (HPH)}

Selection conditions. Complete medium supplemented with 10 to $400 \mu \mathrm{g} / \mathrm{ml}$ hygromycin-B.

Basis for selection. Hygromycin-B is an aminocyclitol that inhibits protein synthesis by disrupting translocation and promoting mistranslation. The $H P H$ gene (isolated from E. coli plasmid pJR225; Gritz and Davies, 1983) detoxifies hygromycin-B by phosphorylation.

Comments. While the level of hygromycin-B needed for selection can vary from 10 to $400 \mu \mathrm{g} / \mathrm{ml}$, many cell lines require $200 \mu \mathrm{g} / \mathrm{ml}$.

References. Gritz and Davies (1983); Palmer et al. (1987).

\section{Puromycin- $N$-acetyl transferase (PAC, puro)}

Selection conditions. Complete medium supplemented with 0.5 to $10 \mu \mathrm{g} / \mathrm{ml}$ puromycin.

Basis for selection. Puromycin inhibits protein synthesis. The gene encodes an enzyme for the inactivation of puromycin by acetylation.

Comment. This selectable marker is comparable to neo in selection efficiency. Many cell types select well at $2.0 \mu \mathrm{g} / \mathrm{ml}$.

Selection of Transfected Mammalian Cells 9.5.8
Reference. de la Luna et al. (1988). 
Thymidine kinase (TK)

Selection conditions. Forward $\left(\mathrm{TK}^{-}\right.$to $\left.\mathrm{TK}^{+}\right)$: Complete medium supplemented with $100 \mu \mathrm{M}$ hypoxanthine, $0.4 \mu \mathrm{M}$ aminopterin, $16 \mu \mathrm{M}$ thymidine, and $3 \mu \mathrm{M}$ glycine (HAT medium).

Basis for selection. Under normal growth conditions, cells do not need thymidine kinase, as the usual means for synthesizing dTTP is through dCDP. Selection of $\mathrm{TK}^{+}$cells in HAT medium is primarily due to the presence of aminopterin, which blocks the formation of dTDP from dCDP. Cells therefore need to synthesize dTTP from thymidine, a pathway that requires TK.

Comments. Thymidine kinase is widely used in mammalian cell culture because both forward and reverse selection conditions exist. Unlike markers such as ADA and DHFR, however, it is not possible to select for variable levels of TK, so the gene cannot be used for amplification. Like ADA and DHFR, most mammalian cell lines express TK, removing the possibility of using the marker in those lines unless BrdU is used to select a $\mathrm{TK}^{-}$mutant.

Reference. Littlefield (1964).

\section{Xanthine-guanine phosphoribosyltransferase (XGPRT, gpt)}

Selection conditions. Medium containing dialyzed fetal bovine serum, $250 \mu \mathrm{g} / \mathrm{ml}$ xanthine, $15 \mu \mathrm{g} / \mathrm{ml}$ hypoxanthine, $10 \mu \mathrm{g} / \mathrm{ml}$ thymidine, $2 \mu \mathrm{g} / \mathrm{ml}$ aminopterin, $25 \mu \mathrm{g} / \mathrm{ml}$ mycophenolic acid, and $150 \mu \mathrm{g} / \mathrm{ml} \mathrm{L}$-glutamine.

Basis for selection. Aminopterin and mycophenolic acid both block the de novo pathway for synthesis of GMP. Expression of XGPRT allows cells to produce GMP from xanthine, allowing growth on medium that contains xanthine but not guanine. It is therefore necessary for selection to use dialyzed fetal bovine serum and a medium that does not contain guanine.

Comments. XGPRT is a bacterial enzyme that does not have a mammalian homolog, allowing XGPRT to function as a dominant selectable marker in mammalian cells. The amount of mycophenolic acid necessary for selection varies with cell type and can be determined by titration in the absence and presence of guanine.

Reference. Mulligan and Berg (1981).

\section{Negative Selection}

\section{Cytosine deaminase (CDA, CD)}

Selection conditions. Complete medium supplemented with 50 to $250 \mu \mathrm{g} / \mathrm{ml} \mathrm{5-}$ fluorocytosine.

Basis for selection. Cytosine deaminase converts the 5-fluorocytosine to 5-fluorouracil, resulting in inhibition of proliferation.

Comment. If the CD-positive cells are a minority $(<1 \%)$, there is no detectable bystander killing; however, if the majority of cells contain $\mathrm{CD}$, then $\mathrm{CD}$-negative cells survive only at higher dilutions ( $\leq 10,000$ plated cells per $100-\mathrm{mm}$ plate).

Reference. Mullen et al. (1992).

Diptheria toxin (DT)

Selection conditions. The expression of the diptheria toxin gene is itself toxic.

Introduction of

DNA into

Mammalian

Cells

9.5.9 
Basis for selection. Diptheria toxin inhibits protein synthesis by the NAD-dependent ADP-ribosylation of elongation factor 2.

Comment. Since this selection is not conditional, this marker is only useful for elimination of cells expressing DT. Therefore, stable cell lines expressing DT cannot be isolated. It has been used as a substitution for HSV-TK for homologous recombination or for the elimination of tissues in transgenic animals.

Reference. Yagi et al. (1990).

Herpes simplex virus thymidine kinase (HSV-TK), + to -

Selection conditions. Complete medium supplemented with $2 \mu \mathrm{M}$ ganciclovir $\{9-[(1,3-$ dihydroxy-2-propoxy)methyl]guanine; DHPG $\}$ or $0.2 \mu \mathrm{M}$ FIAU [1-(2'-deoxy-2'-fluoro$1-\beta$-D-arabinofuranosyl-5-iodo)uracil].

Basis for selection. The selection drugs (ganciclovir or FIAU) are nucleoside analogs and are phosphorylated by the $H S V-T K$ gene. This phosphorylation leads to incorporation of the drug into the growing DNA chain during $S$ phase, and subsequent cell death. Although the related compound 9-[(2-hydroxyethoxy)methyl]guanine (acyclovir) causes chain termination, ganciclovir does not. These drugs are $\sim 1000$-fold poorer substrates for mammalian TK and are thus not harmful to non-HSV-TK-containing cells at these concentrations.

Comment. This system has been widely used for increasing the selection of homologous recombinants by including the HSV-TK gene outside the regions of homology (see UNIT 23.1). It can be used for selection of recombination after Cre recombinase expression if the $T K$ is placed between the lox sites.

References. Cheng et al. (1983); Staschke et al. (1994).

\section{Cloning cylinders}

Glass or metal cloning cylinders can be purchased (e.g., stainless steel Penicylinders, Fisher). Place clean cylinders, well rinsed in distilled water, in $95 \%$ ethanol. Sterilize by flaming immediately prior to use. Alternatively, disposable cloning cylinders can be prepared by cutting off the fat end of a $200-\mu l$ pipettor tip $\sim 1 \mathrm{~cm}$ from the large opening with a razor blade. Sterilize by autoclaving.

\section{COMMENTARY}

\section{Background Information}

Isolation of a cell line stably transfected with DNA encoding a particular gene is dependent on a selection strategy that affords the transfected cell a growth advantage in culture. To implement this approach, an antibioticresistance marker is transfected into cells along with the gene of interest. The antibioticresistance genes allow the transfected cells to survive in a culture containing the corresponding antibiotic, while cells that do not have that marker stably integrated into their genome perish after several weeks in culture.

Thymidine kinase $(T K)$ was the first gene to be extensively used for selection in mammalian cells. The use of 5-bromodeoxyuridine (BrdU) to provide an easy selection for $\mathrm{TK}^{-}$ cells resulted in establishment of several such lines, one of which was used to demonstrate that calcium phosphate-mediated transfection of a viral $T K$ gene could lead to establishment of $\mathrm{TK}^{+}$cells that contained a stably integrated copy of the viral gene (Wigler et al., 1977). This advance made it possible to introduce foreign genes into mammalian cells by transfecting them with a TK-expressing plasmid (cotransfection; Perucho et al., 1980; Robins et al., 1981).

The difficulty with using TK as a selectable marker is that mammalian cells are $\mathrm{TK}^{+}$and only a very low level of TK expression is needed for cells to survive in HAT medium, necessitating the use of a $\mathrm{TK}^{-}$parental cell type. This motivated the development of vectors 
that express bacterial drug resistance genes in mammalian cells. These genes can act as dominant selectable markers in virtually every mammalian cell type, as no endogenous mammalian gene can confer resistance. Four commonly used genes of this sort are $A P H$ (commonly referred to as neo), XGPRT (gpt), $H P H$, and puro (see Basic Protocol 2). These genes allow introduction of exogenous DNA into any cell that can be transfected.

While bacterial-derived markers allow facile introduction of genes into mammalian cells, they do not allow selection over a wide range of selective conditions. The ability to select for expression over a greater than thousand-fold range in drug concentration provides the ability to overexpress newly introduced genes. Mammalian cells that achieve increased resistance to a drug generally have increased the copy number of the gene conferring drug resistance, a process termed amplification. Any gene that is cotransfected with the marker gene will integrate near that gene, and will thus also become amplified. Amplification of the DHFR gene through use of increasing amounts of the drug methotrexate has been widely used to overexpress exogenous genes. The adenosine deaminase $(A D A)$ gene can also be amplified by using increasing concentrations of 2'-deoxycoformycin (dCF; Kaufman et al., 1986).

\section{Critical Parameters}

\section{General considerations}

The efficiency with which the parental cell line can be transfected and the degree of expression of the selective marker determine the success of an attempt to produce stable cell lines. As well, it is important to carefully consider whether the parental cell line is appropriate to the final goal of the research. At least 1 month is required to prepare stable cell lines, and many researchers have reached the end of that time realizing that the resultant line would be much more useful if the initial transfection had been planned more carefully. For example, if stable cell lines are being produced to analyze how a promoter is induced during differentiation, a parental line should be used that differentiates well, and an internal reference promoter might be included in the initial transfection.

In order to produce stable cell lines, it is obviously necessary that the selective marker be expressed at a level adequate to detoxify the selective drug. This is most easily accomplished using $T K$ as a marker when transfecting $\mathrm{TK}^{-}$ cells, or when using any bacterial gene $(A P H$, $X G P R T$, or $H P H$, or puro) as a dominant selectable marker. This is because the recipient cells contain no endogenous gene that detoxifies the selective drugs, so a small level of synthesis from the transfected gene will give the transfected cells a significant advantage over the parental cells. This is much harder to accomplish when using DHFR or ADA to transfect a normal cell type. Mammalian cells can have fairly high endogenous levels of ADA and DHFR, and therefore extremely high levels of expression from the transfected gene may be necessary to enable the recipient cells to grow under selective conditions. While it has proven possible to select stable transfectants of normal cells using both ADA and DHFR, it is difficult. If these markers are used, a parental line that is ADA- or DHFRdeficient greatly increases the yield of transfectants, as a much lower level of selection can be used. If amplification of the transfected genes is desired, use of the deficient lines is recommended, as there is no risk of amplifying the endogenous gene when the selective pressure is applied.

If a cell line cannot be efficiently transfected, it can prove extremely difficult to select stable cell lines using any selective marker. It is essential, then, to have a protocol that provides efficient transfection of the parental cell type. Optimization of transfection conditions for transient expression (see introduction to Section I) usually produces conditions that work well for production of stable cell lines. The exception to this is DEAE-dextran-mediated transfection, which generally works less efficiently for production of stable cell lines than either calcium phosphate transfection or electroporation even with a cell type that is transfected efficiently by DEAE-dextran as judged at transient times after transfection.

Finally, when setting up a transfection to produce stable lines, it is important to remember that the results of the transfection will not be known for two weeks. Having one dish of a transfection fail can therefore be extremely frustrating if a duplicate dish was not done. Many investigators therefore do transfections in duplicate or triplicate to ensure at least one set of successful transfections when trying to produce a particular stable cell line.

\section{Anticipated Results}

Approximately $10^{3}$ colonies can be produced by transfecting $1 \mu \mathrm{g}$ of a plasmid expressing a selective marker into $10^{6}$ cells from a highly transfectable cell line (e.g., HeLa,
Introduction of

DNA into

Mammalian

Cells

\subsubsection{1}

Supplement 86 
CHO DUKX, NIH 3T3). Approximately 10\% of these will be large, vigorously growing colonies that can readily be expanded into cell lines. The precise number of stable transfectants to be expected can vary dramatically depending on the cell type and the efficiency with which the marker gene is expressed.

\section{Time Considerations}

It takes 1 day to set up the DNA and perform the initial transfections. The transfected cells should double twice before being split into selective medium, and after being placed in selective medium generally take 10 to 12 days to form colonies. Another week or so is required to expand the colonies into cell lines. The entire procedure takes $\sim 1$ month.

\section{Literature Cited}

Cheng, Y.C., Huang, E.S., Lin, J.C., Mar, E.C., Pagano, J.S., Dutschman, G.E., and Grill, S.P. 1983. Unique spectrum of activity of 9-[(1,3dihydroxy-2-propoxy)methyl]guanine against herpesviruses in vitro and its mode of action against herpes simplex virus type 1. Proc. Natl. Acad. Sci. U.S.A. 80:2767-2770.

de la Luna, S., Soria, I., Pulido, D., Ortin, J., and Jimenez, A. 1988. Efficient transformation of mammalian cells with constructs containing a puromycin-resistance marker. Gene 62:121126.

Fukushige, S. and Sauer, B. 1992. Genomic targeting with a positive- selection lox integration vector allows highly reproducible gene expression in mammalian cells. Proc. Natl. Acad. Sci. U.S.A. 89:7905-7909.

Gossen, M. and Bujard, H. 1992. Tight control of gene expression in mammalian cells by tetracycline-responsive promoters. Proc. Natl. Acad. Sci. U.S.A. 89:5547-5551.

Gossen, M., Freundlieb, S., Bender, G., Muller, G., Hillen, W., and Bujard, H. 1995. Transcriptional activation by tetracyclines in mammalian cells. Science 268:1766-1769.

Gritz, L. and Davies, J. 1983. Plasmid-encoded hygromycin-B resistance: The sequence of hygromycin-B-phosphotransferase gene and its expression in E. coli and S. cerevisiae. Gene 25:179-188.

Hartman, S.C. and Mulligan, R.C. 1988. Two dominant-acting selectable markers for gene transfer studies in mammalian cells. Proc. Natl. Acad. Sci. U.S.A. 85:8047-8051.

Kaufman, R.J., Murtha, P., Ingolia, D.E., Yeung, C-Y., and Kellems, R.E. 1986. Selection and amplification of heterologous genes encoding adenosine deaminase in mammalian cells. Proc. Natl. Acad. Sci. U.S.A. 83:3136-3140.

Kozak, M. 1989. The scanning model for translation: An update. J. Cell Biol. 108:229-241.

Littlefield, J.W. 1964. Selection of hybrids from matings of fibroblasts in vitro and their presumed recombinants. Science 145:709-710.
Mullen, C.A., Kilstrup, M., and Blaese, R.M. 1992. Transfer of the bacterial gene for cytosine deaminase to mammalian cells confers lethal sensitivity to 5-fluorocytosine: A negative selection system. Proc. Natl. Acad. Sci. U.S.A. 89:33-37.

Mulligan, R.C. and Berg, P. 1981. Selection for animal cells that express the $E$. coli gene coding for xanthine-guanine phosphoribosyltransferase. Proc. Natl. Acad. Sci. U.S.A. 78:20722076.

Mulsant, P., Gatignol, A., Dalens, M., and Tiraby, G. 1988. Phleomycin resistance as a dominant selectable marker in CHO cells. Somatic Cell Mol. Genet. 14:243-252.

No, D., Yao, T.P., and Evans, R.M. 1996. Ecdysoneinducible gene expression in mammalian cells and transgenic mice. Proc. Natl. Acad. Sci. U.S.A. 93:3346-3351.

Palmer, T.D., Hock, R.A., Osborne, W.R.A., and Miller, A.D. 1987. Efficient retrovirus-mediated transfer and expression of a human adenosine deaminase gene in diploid skin fibroblasts from an adenosine-deficient human. Proc. Natl. Acad. Sci. U.S.A. 84:1055-1059.

Perucho, M., Hanahan, D., and Wigler, M. 1980. Genetic and physical linkage of exogenous sequences in transformed cells. Cell 22:309317.

Robertson, E.J. 1987. Embryo-derived stem cell lines. In Teratocarcinomas and Embryonic Stem Cells: A Practical Approach (E.J. Robertson, ed.) pp. 71-112. IRL Press, Oxford and New York.

Robins, D.M., Ripley, S., Henderson, A.S., and Axel, R. 1981. Transforming DNA integrates into the host chromosome. Cell 23:29-39.

Simonsen, C.C. and Levinson, A.D. 1983. Isolation and expression of an altered mouse dihydrofolate reductase cDNA. Proc. Natl. Acad. Sci. U.S.A. 80:2495-2499.

Southern, P.J. and Berg, P. 1982. Transformation of mammalian cells to antibiotic resistance with a bacterial gene under control of the SV40 early region promoter. J. Mol. Appl. Gen. 1:327341.

Staschke, K.A., Colacino, J.M., Mabry, T.E., and Jones, C.D. 1994. The in vitro anti-hepatitis B virus activity of FIAU [1-(2' -deoxy-2'-fluoro$1-\beta$-D-arabinofuranosyl-5-iodo)uracil] is selective, reversible, and determined, at least in part, by the host cell. Antivir. Res. 23:45-61.

Sugiyama, M., Thompson, C.J., Kumagai, T., Suzuki, K., Deblaere, R., Villarroel, R., and Davies, J. 1994. Characterisation by molecular cloning of two genes from Streptomyces verticillus encoding resistance to bleomycin. Gene 151:11-16.

Wei, K. and Huber, B.E. 1996. Cytosine deaminase gene as a positive selection marker. J. Biol. Chem. 271:3812-3816.

Wigler, M., Silverstein, S., Lee, L- S., Pellicer, A., Cheng, Y.- C., and Axel, R. 1977. Transfer of purified herpes virus thymidine kinase gene to cultured mouse cells. Cell 11:223-232. 
Yagi, T., Ikawa, Y., Yoshida, K., Shigetani, Y., Takeda, N., Mabuchi, I., Yamamoto, T., and Aizawa, S. 1990. Homologous recombination at c-fyn locus of mouse embryonic stem cells with use of diphtheria toxin A-fragment gene in negative selection. Proc. Natl. Acad. Sci. U.S.A. 87:9918-9922.

Introduction of

DNA into

Mammalian

Cells

9.5.13 